\title{
Calcium current improves coincidence detection of the LIF model
}

\author{
Yansong Chua ${ }^{1,2^{*}}$, Moritz Helias ${ }^{1}$, Abigail Morrison ${ }^{1,2,3}$ \\ From The Twenty Third Annual Computational Neuroscience Meeting: CNS*2014 \\ Québec City, Canada. 26-31 July 2014
}

Dendritic spikes are known to improve efficacy of synaptic inputs in causing action potentials [1]. The calcium spike at distal apical dendrites of layer 5 pyramidal neurons has been observed in-vitro and argued to support the propagation of synaptic inputs from distal tufts to the soma [2]. When combined with a back-propagating action potential, a smaller distal current is sufficient to trigger a calcium spike [3]. Recently, it has also been shown in-vivo that dendritic spikes contribute to the neuronal activity $[4,5]$.

Calcium spikes have been modeled in multi-compartment point neuron models using first order kinetics [6]. Here we show that calcium spikes, in the regime of large synchronous inputs on top of a background of weakly fluctuating synaptic noise can be well approximated by a threshold-triggered current of fixed waveform. The exact contribution of the calcium spike to the somatic membrane potential can then be analytically derived. Accurate predictions are only obtained if correlations between the membrane potential and synaptic conductances are taken into account [7].

Comparing neuron models with and without calcium dynamics, we find that the calcium current increases the sensitivity of the neuron's spiking response to sufficiently large coincident input. In numerical simulations carried out with NEST [8], we investigate the effect of the jitter of close to synchronous inputs on the probability to elicit a calcium spike. With increased jitter, fewer calcium spikes are elicited and their average amplitude decreases.
Modeling for the Human Brain", the Jülich Aachen Research Alliance (JARA), the Junior Professor Program of Baden-Württemberg, the HGF Nachwuchsgruppe VH-NG-1028: Theory of multi-scale neuronal networks, and the Initiative and Networking Fund of the Helmholtz Association.

\section{Authors' details}

'Institute of Neuroscience and Medicine (INM-6) and Institute for Advanced Simulation (IAS-6), Juelich Forschungszentrum, Juelich, Germany. ${ }^{2}$ Bernstein Center Freiburg, Albert-Ludwigs University, Freiburg im Breisgau, Germany. ${ }^{3}$ Institute for Cognitive Neuroscience, Faculty of Psychology, Ruhr University of Bochum, Bochum, Germany.

Published: 21 July 2014

\section{References}

1. Williams SR, Stuart GJ: Dependence of EPSP Efficacy on Synapse Location in Neocortical Pyramidal Neurons. Science 2002, 295:1907-1910.

2. Larkum ME, Zhu JJ, Sakmann B: Dendritic mechanisms underlying the coupling of the dendritic with the axonal action potential initiation zone of adult rat layer 5 pyramidal neurons. Journal of Physiology 2001, 533:447-466.

3. Larkum ME, Zhu JJ, Sakmann B: A new cellular mechanism for coupling inputs arriving at different cortical layers. Nature 1999, 398:338-341.

4. Smith SL, Smith IT, Branco T, Häusser M: Dendritic spikes enhance stimulus selectivity in cortical neurons in vivo. Nature 2013, 503:115-120.

5. Sivyer B, Williams SR: Direction selectivity is computed by active dendritic integration in retinal ganglion cells. Nature Neuroscience 2013, 16:1848-1856.

6. Larkum ME, Senn W, Lüscher HR: Top-down Dendritic Input Increases the Gain of Layer 5 Pyramidal Neurons. Cerebral Cortex 2004, 14:1059-1070.

7. Richardson MJE, Gerstner W: Synaptic Shot Noise and Conductance Fluctuations Affect the Membrane Voltage with Equal Significance. Neural Computation 2005, 17:923-947.

8. Gewaltig M, Diesmann M: NEST (Neural Simulation Tool). Scholarpedia 2007, 2(4):1430.

doi:10.1186/1471-2202-15-S1-P86

Cite this article as: Chua et al.: Calcium current improves coincidence detection of the LIF model. BMC Neuroscience 2014 15(Suppl 1):P86.

\section{Acknowledgements}

Partially supported by the Helmholtz Alliance on Systems Biology, the Helmholtz Association in the Portfolio theme "Supercomputing and

\footnotetext{
* Correspondence: y.chua@fz-juelich.de

1 Institute of Neuroscience and Medicine (INM-6) and Institute for Advanced

Simulation (IAS-6), Juelich Forschungszentrum, Juelich, Germany

Full list of author information is available at the end of the article
} 